\title{
Design and characterisation of SRS filtering optical fibre for pulsed fibre laser beam delivery
}

Natasha Vukovic, Jaclyn S. Chan, Philip M. Gorman, Marco N. Petrovich, John R. Hayes, et al.

Natasha Vukovic, Jaclyn S. Chan, Philip M. Gorman, Marco N. Petrovich, John R. Hayes, Andy Malinowski, Christophe A. Codemard, Michalis N. Zervas, "Design and characterisation of SRS filtering optical fibre for pulsed fibre laser beam delivery," Proc. SPIE 10083, Fiber Lasers XIV: Technology and Systems, 1008312 (22 February 2017); doi: 10.1117/12.2251980

SPIE. Event: SPIE LASE, 2017, San Francisco, California, United States 


\title{
Design and characterisation of SRS filtering optical fibre for pulsed fibre laser beam delivery
}

\author{
Natasha Vukovic ${ }^{\mathrm{a}^{*}}$, Jaclyn S. Chan ${ }^{\mathrm{a}}$, Philip M. Gorman ${ }^{\mathrm{b}}$, Marco N. Petrovich ${ }^{\mathrm{a}}$, John R. Hayes ${ }^{\mathrm{a}}$, \\ Andy Malinowski ${ }^{\mathrm{b}}$, Christophe A. Codemard ${ }^{\mathrm{b}}$, and Michalis N. Zervas ${ }^{\mathrm{a}, \mathrm{b}}$ \\ ${ }^{a}$ Optoelectronics Research Centre, University of Southampton, Southampton SO17 1BJ, UK; \\ ${ }^{\mathrm{b}}$ Advanced Laser Laboratory, SPI Lasers UK Ltd, Southampton SO30 2QU, UK
}

\begin{abstract}
The high performance of fibre lasers is largely due to the outstanding characteristics of fibres as an active medium. However, there is a need to overcome some limits at high optical powers which are imposed by the fibre design. We report on a design and fabrication of a stimulated Raman scattering (SRS) filtering fibre for high average or high peak optical power delivery applications. The fibre geometry is based on the series of circularly arranged high index rod resonators embedded in the silica cladding. The operation principle relies on the resonant coupling of the core and resonators modes. The fabricated fibre demonstrated wide transmission window and filtering of SRS from the output spectra (with the extinction which exceeds $20 \mathrm{~dB}$ at the Raman Stokes wavelength), robustness for bending and high output beam quality. The fibre has been tested as a beam delivery fibre of a commercial pulsed fibre laser system in order to identify filtering performance and its limitations.
\end{abstract}

Keywords: Stimulated Raman Scattering, SRS filtering fibre, high power fibre laser, beam delivery

\section{INTRODUCTION}

Over the last decade fibre lasers have developed from lab experiment to be mainstream industrial laser sources thanks to their superior performances, including high-power, superior beam quality and stability, high brightness, as well as efficient thermal management and elimination of thermal lensing ${ }^{1}$. The expansion and progress of this technology has been driven by improved and novel fibre designs. However, the increase of optical power leads to some unwanted nonlinear effects, such as self-focusing, self-phase modulation, stimulated Brillouin scattering (SBS), and stimulated Raman scattering ${ }^{1,2}$. Although the ultimate limit on the laser performance is set by self-focusing, it is considered that self-phase modulation is a limiting effect for short pulses $(<0.5 \mathrm{~ns})$, SBS is detrimental effect for narrow linewidth, long pulses ( $>2 \mathrm{~ns}$ ), while SRS is a limiting nonlinearity for spectral broadband signals in any temporal regime ${ }^{2,3}$. Stimulated Raman scattering is a combination of Raman scattering with stimulated emission, which leads to Raman amplification. At high optical power density, SRS leads to scattering (and energy transfer) of pump photons from the optical signal into first Stokes photons, and then in a cascading fashion this leads to generating second and higher order Stokes ${ }^{4}$, which limits the available output power in fibre lasers at a given signal wavelength. Because SRS is a nearly instantaneous process, it is considered the main obstacle for power scaling of broadband pulsed fibre systems, and in particular when singlemode core fibres $(\mathrm{V}<2.4)$ are employed, in which case the SRS threshold is significantly reduced ${ }^{5}$. In the continuous wave $(\mathrm{CW})$ regime, apart from transverse mode instability, stimulated Raman scattering is considered to be the most limiting nonlinear effect of practical multi-kW class high power fibre lasers with beam delivery. Therefore, a suppression of SRS remains an important challenge.

To date, a number of various techniques and designs have been considered for the suppression of detrimental SRS, including employing long-period gratings to attenuate the scattered beam ${ }^{6}$, or using special fibres, such as based on resonant rings design ${ }^{5,7-9}$ or photonic bandgap fibres ${ }^{10-12}$. However, in those designs, despite the Raman filtering due to the resonant coupling between core and cladding modes, the fibre bending must be carefully controlled, or only short fibre lengths are permitted. In addition, in W-types fibres with high index rings, it has been observed that different proposed designs have limitations due to undesired coupling to ring modes, which reduce dramatically the transmission window and induce very high tolerance for the fibre's structural parameters, even in the case of unbent fibre. Furthermore, the SRS suppression has been demonstrated on the basis of wavelength dependent loss and bending $\operatorname{loss}^{13,14}$, but this approach suffers from introducing significant amount of loss on the signal wavelength, too. Therefore, different strategies must be used to improve the fibre performance to better address the SRS filtering aspect.

*ntv@orc.soton.ac.uk; phone: +44(0)2380597230

Fiber Lasers XIV: Technology and Systems, edited by Craig A. Robin, Ingmar Hartl, Proc. of SPIE Vol. 10083, 1008312 - (C) 2017 SPIE · CCC code: 0277-786X/17/\$18 - doi: 10.1117/12.2251980 
In this paper we demonstrate a novel fibre design for the efficient suppression of SRS. The fibre structure is based on a $\mathrm{W}$-type fibre with high index rings, however high index rods resonators are added in the cladding instead. In this case, the series of circularly arranged rods break the azimuthal symmetry of the resonant rings, so that no rod-mode crosses the dispersion curve of the fundamental mode near the designed operating wavelength. The basic mechanism relies on the index matching and resonant coupling between core and cladding modes. We show that this fibre has advantages in comparison to the resonant ring designs, and that it is suitable for applications of high power pulsed laser beam delivery applications.

\section{SRS FILTERING FIBRE MODELLING AND DESIGN}

In order to introduce substantial loss between the desired signal and parasitic SRS wavelengths, the proposed fibre design combines wavelength dependent mode delocalization and spatially dependent loss mechanism. The principle of operation is shown schematically in Figure 1. The mode delocalization is achieved through multi-stage resonant coupling between the core modes and appropriately designed high-index rods placed optimally in the fibre cladding. This results in a fibre fundamental mode being highly delocalized and spread-out in the composite cladding through successive couplings in the high index rods. At the resonant (Raman) wavelength, at which the effective index of the core and cladding are matched, light in the core can efficiently couple to leaky cladding modes. In contrast, the fundamental mode is not resonant with the surrounding rods and remains highly localized in the core, at the signal wavelength. In this way the wavelength dependent coupling is translated into wavelength-dependent spatial distribution. The spatially dependent loss then can be achieved by introducing a high-index leaky structure in the outer cladding region with or without additional losses.

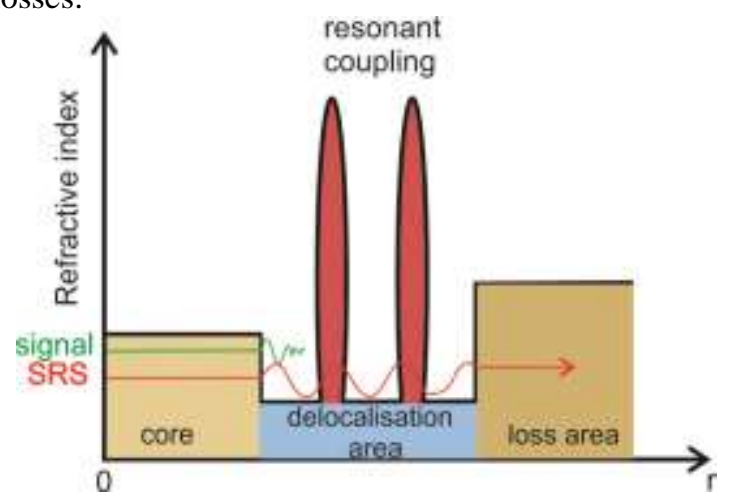

Figure 1. Concept of SRS filtering due to resonant coupling between core and cladding modes.

The proposed SRS mitigating fibre structure comprises standard silica core and Ge-doped high index rods embedded in fluorine-doped tubes in a hexagonal lattice in the pure silica outer leaky layer, as shown in Figure 2(a). The refractive index profile of the structure is given in Figure 2(b). The parameters of the fibre are: silica core diameter $15 \mu \mathrm{m}$, the Gedoped rods are with parabolic profile raised index $\Delta \mathrm{n}_{\mathrm{Ge}}=2 \%$ (obtained from the measured refractive index profile) and depressed cladding with $\Delta \mathrm{n}_{\text {core }}=-0.15 \%$. The ratio of fluorinated cladding diameter $\left(\mathrm{D}_{\mathrm{F}}\right)$ and Ge-rod core diameter $\left(\mathrm{D}_{\mathrm{Ge}}\right)$ is 2.394 . The fibre estimated core NA is $\sim 0.07$.
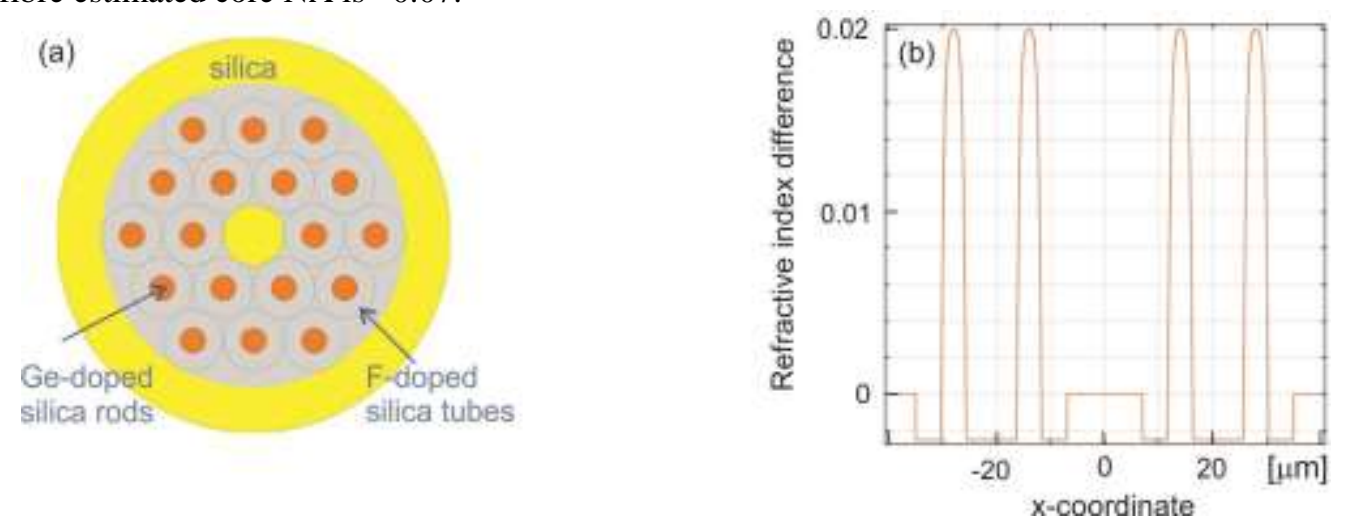

Figure 2. (a) Cross section of a SRS filtering fibre design. (b) Refractive index profile. 
We used a full vector modal solver based on Finite Element Method (implementation based on COMSOL Multiphysics) to model the SRS mitigating fibre. The extensive modelling focused on fulfilling the rigorous specification, which includes SRS filtering efficiency, overall loss, bend loss, fibre modality, and mode field diameter, taking into account the measured refractive index of Ge rods (using a Intertronics Fiber Analyzer). In particular, the specifications included: negligible bend loss at $10 \mathrm{~cm}$ radius, negligible fundamental mode loss (less than $0.01 \mathrm{~dB} / \mathrm{m}$ ) at the signal wavelength, greater than $100 \mathrm{~dB}$ total loss at the Raman wavelength for the SRS suppression in high average (kW level) or high peak $(>200 \mathrm{~kW})$ optical power delivery applications. Importantly, the model also considered the actual refractive index distribution of the Ge-doped rods within the fibre cladding.

The numerical modelling was used to identify the parameters that enable phase-matching between the core mode and high order rod modes at Raman wavelength, and consequently to enable efficient SRS filtering, whilst keeping the loss at signal wavelength low. The parameters which satisfy the phase matching condition between the core $\mathrm{LP}_{01}$ mode and high order Ge-rods modes are: Ge-rod core diameter is $6.27 \mu \mathrm{m}$ and the fluorinated tube diameter is $15 \mu \mathrm{m}$. This fixes the rod core-to-core distance to $\Lambda=15 \mu \mathrm{m}$ (such that $\mathrm{D}_{\mathrm{F}} / \Lambda=1$ ). As shown in Figure 3(a), these parameters ensure phase matching and efficient coupling of the fundamental core mode $\mathrm{LP}_{01}$ to the $\mathrm{LP}_{21}$ and $\mathrm{LP}_{02}$ of the rod modes at the Raman Stokes wavelength. At the signal wavelength, on the other hand, fibre core and high index rods remain phase mismatched and therefore no substantial coupling (and power delocalization) takes place. The dispersion curves in Figure 3(a) are based on modelling of the two different step index fibre structures; first is comprised of the silica core and fluorinated silica cladding, and second comprises step index fibre with Ge-doped silica core and fluorinated silica cladding. It can be observed that index matching between core and cladding modes is observed at Raman wavelength $(\sim 1125 \mathrm{~nm})$, whilst the signal operating wavelength should be further from the intersection of effective index curves intersection $(\sim 1070 \mathrm{~nm})$. Further sensitivity analysis proved the robustness of the design and no performance variations for bending down to $10 \mathrm{~cm}$ bending radii, as shown in Figure 3(b). It is shown that the design parameters result in $<0.01 \mathrm{~dB} / \mathrm{m}$ loss at the signal wavelength and $>100 \mathrm{~dB} / \mathrm{m}$ leakage loss at the SRS wavelength. Moreover, this design exhibits no performance variations with $\pm 200 \mathrm{~nm}$ random shifts in the rod positions in the low index cladding. The modelling suggests that the geometrical parameters would need to be satisfied to within approximately $\pm 10 \%$ tolerance to achieve the required performance.
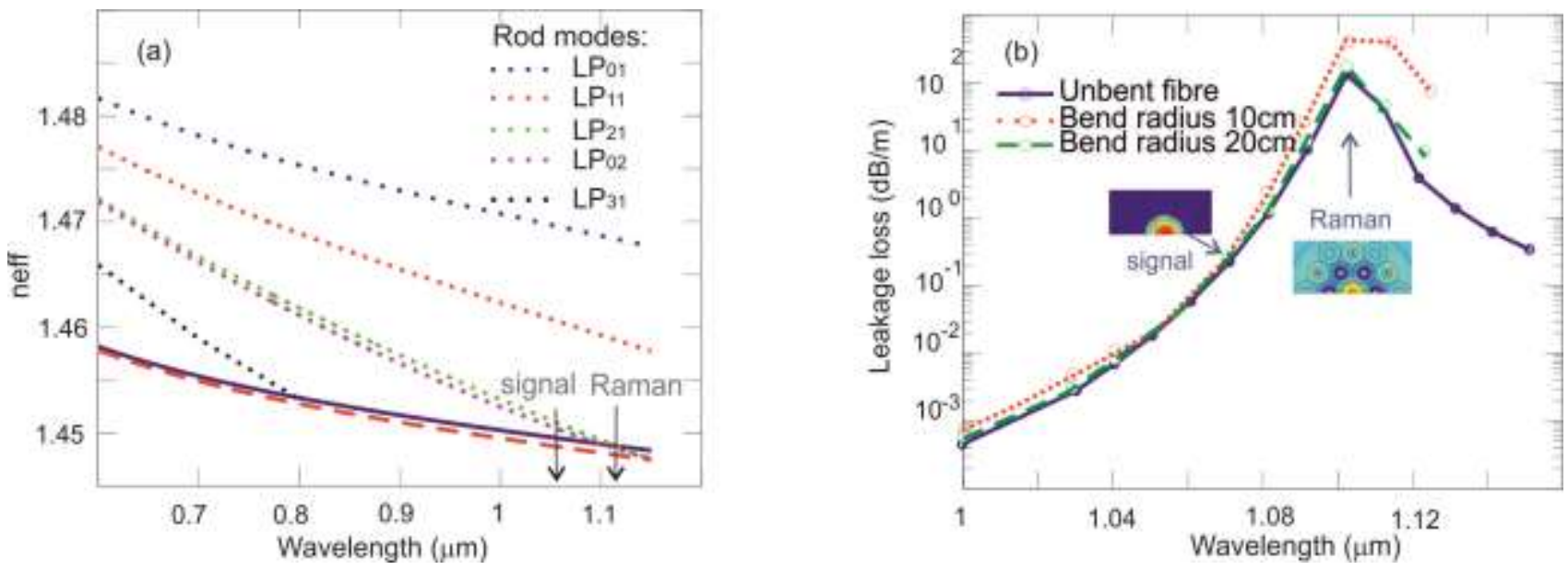

Figure 3. (a) Effective indices of modes as a function of wavelength. Solid line corresponds to the LP01 (blue) and dashed line corresponds to LP11 (red) modes of the silica core modes; dotted lines correspond to the Ge-rod modes; (b) Effect of bend radius on a leakage loss of the SRS suppression fibre. The mode field diameter at $1070 \mathrm{~nm}$ is $\sim 17.5 \mu \mathrm{m}$.

\section{SRS FILTERING FIBRE FABRICATION AND CHARACTERIZATION}

Despite the fabrication challenges related to the stacking and drawing of a complex fibre structure with high germanium content, the fibre has been successfully fabricated, as shown in Figure 4(a). The drawn fibre outer diameter was $260 \mu \mathrm{m}$ and core diameter $16 \mu \mathrm{m}$. The microscopic image of a cross section of the SRS filtering fibre is shown in Figure 4(a). It can be observed that nearly perfect hexagonal structure, and a good agreement with the design parameters, has been achieved. 
Using a high resolution OTDR at 1300nm, operating outside the filtering range, the background loss was measured to be about $\sim 0.032 \mathrm{~dB} / \mathrm{m}$, as shown in the inset of Figure 4(b). Using a broadband light source the cut back measurement was performed. The loss spectra indicates high loss bands in the wavelength range which correspond to the intersection of the effective indices curves shown in Figure 3(a), and the loss in the SRS range is well in excess of 20dB. This result implies an excellent agreement between the prediction obtained from the numerical modelling and the experimental measurements. However, we observed some losses at the signal wavelength which we attributed to an imperfect launch into the fibre core.
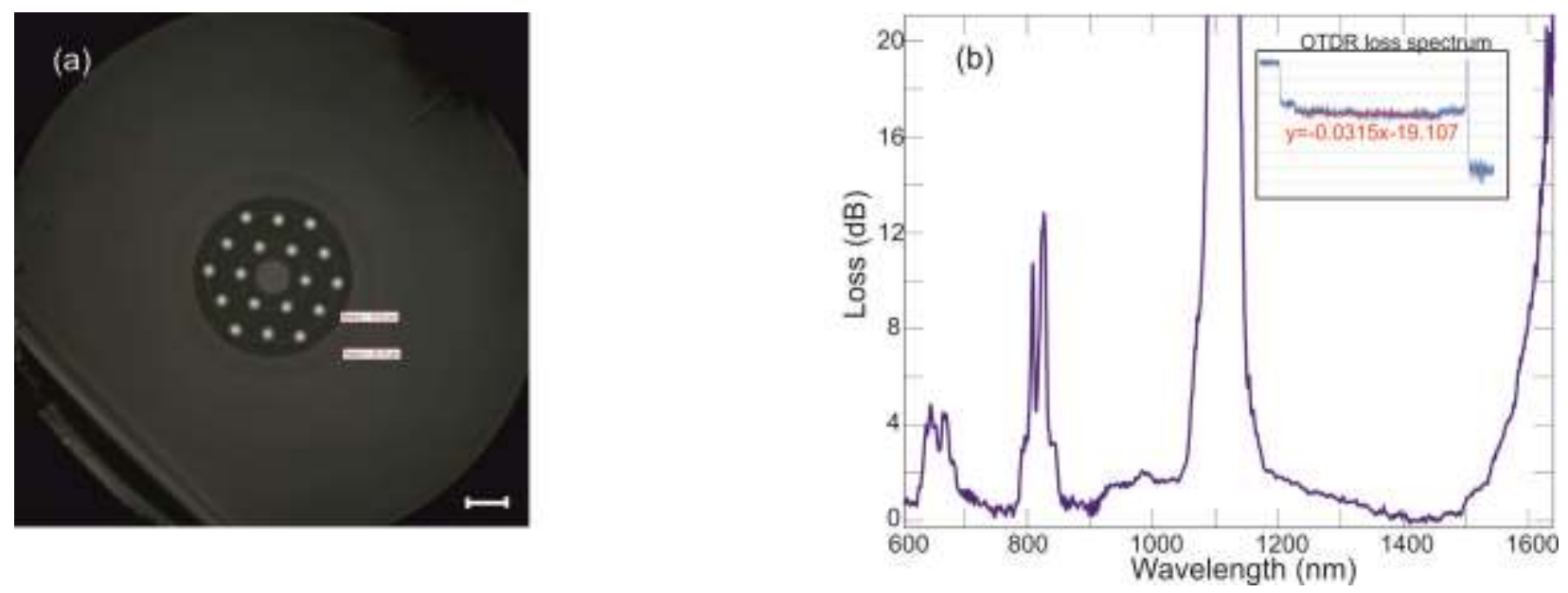

Figure 4. (a) Microscope image of the SRS filtering fibre, scale $=20 \mu \mathrm{m}$. (b) Loss spectrum measurement. Inset: OTDR loss spectra, for the $9.5 \mathrm{~m}$ long SRS suppression fibre (blue), and the corresponding linear fit (dashed red curve).

In addition, the SRS fibre was tested as a beam delivery fibre of a commercial pulsed fibre laser operating at $1 \mathrm{MHz}$ with 2 ns long pulses. The characterization setup is shown in Figure 5. The light is launched to the SRS suppression fibre from a modified G4 fiber laser from SPI laser, which is capable of delivering $35 \mathrm{~kW}$ peak power. The $\sim 1 \mathrm{~m}$ delivery fibre is spliced to the $\sim 25 \mathrm{~cm}$ of a step index fibre with the suitable mode field diameter, so that it can match the SRS suppression fibre. The SRS suppression fibre length was $9.5 \mathrm{~m}$, and it was coiled with $30 \mathrm{~cm}$ and $15 \mathrm{~cm}$ diameter. A short piece of the same step index fibre used at the input of the SRS was added at the end of the SRS fibre, with an additional $0.8 \mathrm{~mm}$ end-cap, made of matched cladding size $(250 \mu \mathrm{m})$ coreless fibre.

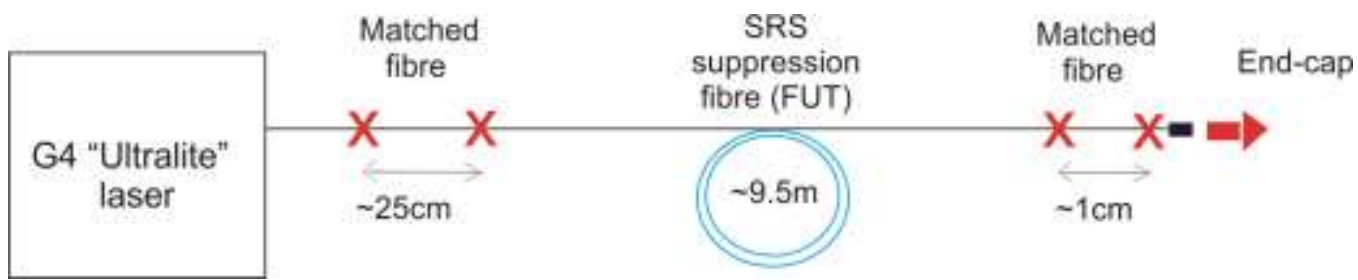

Figure 5. Experimental setup for the pulsed laser application.

The measurement results of the output spectra are shown in Figure 6. The results clearly indicate that the SRS wavelength band is filtered out from the output spectra, as no measurable emission is present above $\sim 1100 \mathrm{~nm}$. In addition, the output beam $\mathrm{M}^{2}$ was measured less than 1.1 (see inset of Figure 6). Some astigmatism due to the anglecleave on the end-cap can be also observed.

In Figure 6, we observed that coiling only slightly impact the transmitted signal peak power. However with the input power increase, the excess peak power that generates the SRS is filtered out as bending slightly modified the position of the spectral filtering edge. 


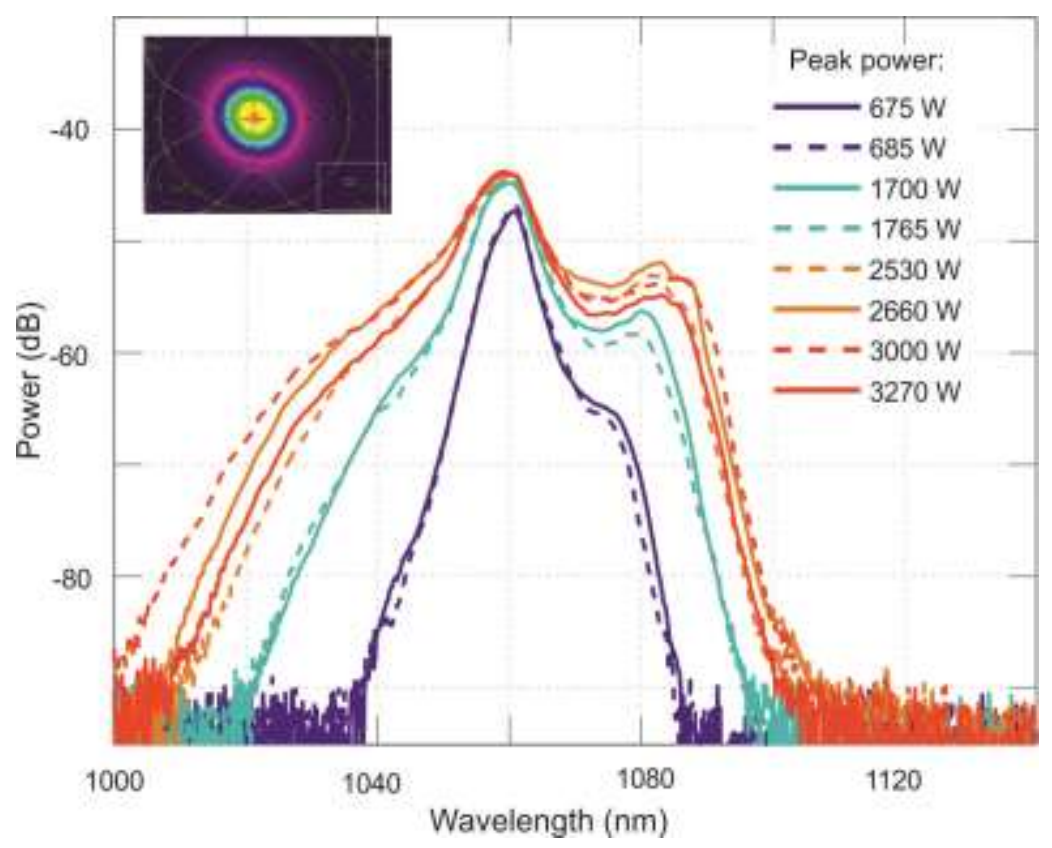

Figure 6. OSA output spectra (resolution $1 \mathrm{~nm}$ ) obtained for the $2 \mathrm{~ns}$ pulses at $1 \mathrm{MHz}$ repetition rate. Solid line: coil diameter $30 \mathrm{~cm}$, dashed line: $15 \mathrm{~cm}$. Inset: Output beam at $\sim 1065 \mathrm{~nm}$ at maximum power.

Furthermore, excess spectral broadening is observed with increased peak powers, which we attribute to enhanced four wave mixing (FWM) process in the SRS filtering fibre. Further tests using a shorter piece of FUT showed that for the same peak power, much narrower spectra are obtained, which confirms the existence of the FWM phase matched bands in the FUT. Additional experiments showed that the FWM process has not degraded the output beam quality and the $\mathrm{M}^{2}$ remained less than 1.1. The strategies for mitigation of FWM, which also include careful design of the fibre effective area and dispersion properties, are still under investigation.

\section{SUMMARY}

We have presented the first demonstration of a novel fibre for the filtering of stimulated Raman scattering for high average or high peak optical power delivery applications. The fibre is based on the series of circularly arranged high index Ge rods embedded in a fluorine doped silica cladding. The functionality of the fibre is based on the combination of the wavelength dependent mode power delocalization and spatially dependent loss mechanism. The key advantage of this novel design is in a reduced sensitivity to bending in comparison to the $\mathrm{W}$-type ring fibres and photonic bandgap fibres counterparts. The proposed fibre is fabricated using stack-and-draw technique. The experimental characterization showed low loss of $\sim 0.032 \mathrm{~dB} / \mathrm{m}$, and the loss extinction in the excess of $20 \mathrm{~dB}$ at the Raman wavelength. The fibre was used experimentally as a delivery fibre for the $2 \mathrm{~ns}$ pulses laser and showed a good output beam quality at the peak powers of up to $\sim 3 \mathrm{~kW}$. Importantly, preliminary results open up the potential for the new class of SRS filtering fibre to find use in high power fibre laser applications. Ongoing research will focus on the means to mitigate the four wave mixing, and to explore designs that would be even easier to fabricate.

\section{REFERENCES}

[1] Zervas, M. N., and Codemard, C. A., "High power fiber lasers: a review," IEEE J. of Sel. Topics in Quantum Electronics 20 (5), 0904123 (2014).

[2] Jauregui, C., Limpert, J., and Tünnermann, A., "High-power fibre lasers," Nat. Photonics 7, 861-867 (2013).

[3] Alkeskjold, T. T., "Large-mode-area Ytterbium-doped fiber amplifier with distributed narrow spectral filtering and reduced bend sensitivity," Opt. Express 17(19), 16394-16405 (2009).

[4] Stolen, R. H., Ippen, E. P., and Tynes, A. R., "Raman oscillations in glass optical waveguide," Appl. Phys. Lett., 20 (62) (1972). 
[5] Kim, J., Dupriez, C., Codemard, C., Nilsson, J., and Sahu, J. K., "Suppression of stimulated Raman scattering in a high power Yb-doped fiber amplifier using a W-type core with fundamental mode cut-off," Opt. Express 14(12), 5103-5113 (2006).

[6] Nodop, D., Jauregui, C., Jansen, F., Limpert, J., and Tünnermann, A., "Suppression of stimulated Raman scattering employing long period gratings in double-clad fibre amplifiers," Opt. Lett. 35, 2982-2984 (2010).

[7] Fini, J. M., Bise, R. T., Yan, M. F., Yablon, A. D., and Wisk, P. W., "Distributed fiber filter based on index-matched coupling between core and cladding," Opt. Express 13(25), 10022-10033 (2005).

[8] Fini, J. M., Mermelstein, M. D., Yan, M. F., Bise, R. T., Wisk, P. W., and Andrejco, M. J., "Distributed suppression of stimulated Raman scattering in an Yb-doped filter-fiber amplifier," Opt. Lett. 31(17), 2550-2552 (2006).

[9] Neves, V. I., and Fernandes, "Modal characteristics for W-type and M-type dielectric profile fibres," Micro.\&Opt. Tech. Lett. 22, 398-405 (1999).

[10] Wei, H., Yan, P., Chen, S., Tong, W., Luo, J., and Ruan, S., “A broadband ultra-low loss all-solid photonic bandgap fiber," OFC 2010.

[11] Kashiwagi, M., Saitoh, K., Takenaga, K., Tanigawa, S., Matsuo, S., and Fujimaki, M., "Effectively single-mode allsolid photonic bandgap fiber with large effective area and low bending loss for compact high-power all-fiber lasers," Opt. Express 20 (14), pp. 15061-15070 (2012).

[12] Gerome, F., Auguste, J. L., and Blondy, J. M., "Design of dispersion-compensating fibers based on a dualconcentric-core photonic crystal fiber," Opt. Lett. 29, 2725 (2004|).

[13] Kuzin, E. A., Beltran-Perez, G., Basurto_Pensado, M. A., Rojas-Laguna, R., Andrade-Lucio, J. A., Torres-Cisneros M., and Alvarado-Mendez E., "Stimulated Raman scattering in a fiber with bending loss," Opt. Commun. 169, 8791 (1999).

[14] Dragic, P. D., "Suppression of first order stimulated Raman scattering in Erbium-doped fiber laser based LIDAR transmitters through induced bending loss," Opt. Commun. 250, 403-410 (2005). 